\title{
The Influence of Angles Kappa and Alpha and Pupil Size on Vision after Implantation of Soleko Evolve and Lucidis Lenses with a "Pure" EDOF Structure
}

\author{
Adam Cywinski ${ }^{1 *}$ \\ ${ }^{1}$ Silesian Eye Treatment Centre, Zory, Poland \\ *Corresponding Author: Adam Cywinski, Silesian Eye Treatment Centre, Zory, Poland; \\ Email: adamcyw@gmail.com
}

Received Date: 03-06-2021; Accepted Date: 23-06-2021; Published Date: 30-06-2021

Copyright $^{\oplus} 2021$ by Cywinski A. All rights reserved. This is an open access article distributed under the terms of the Creative Commons Attribution License, which permits unrestricted use, distribution, and reproduction in any medium, provided the original author and source are credited.

\begin{abstract}
Background: Two models of artificial intraocular lenses with a "pure" EDOF structure, i.e. Soleco Evolve and Lucidis, were used in 36 patients in 43 eyes. Results of postoperative visual function were analysed, like also a correlation between preoperatively measured parameters of pupil size, kappa and alpha angles.

Methods and Findings: Cataract and an unacceptable refractive error mainly hyperopia, were the reasons for the removal of the lens from the eye. High values of angles kappa and alpha or a large pupil were not a disqualifying factor for implantation of these lens models. The beneficial effect of improving vision too far and near distances was achieved in most eyes. The dependence on light intensity and pupil size are the main factors affecting visual acuity too far and near distances. High values of angles alpha and kappa had only a slight, negative effect on the postoperative visual acuity values.

Conclusion: A sufficiently large pupil size determined preoperatively allows for satisfactory visual acuity too far and near distances to be obtained. In hyperopic eyes after refractive lens exchange, the values of visual acuity to far distances were slightly lower than before surgery. This information determines the indication for the use of "pure" EDOF lenses in non-dominant eyes and in patients with a large pupil. In cases of a very large pupil diagnosed preoperatively, vision to near distances without the need for additional correction, obtained in good lighting conditions, is an added value, which seems to be a sufficient advantage over the benefits of monofocal lens implantation.
\end{abstract}




\section{Keywords}

EDOF; Angle Kappa; Angle Alpha; Large Pupil; Evolve, Lucidis

\section{Introduction}

Cataract and an unacceptable refractive error are two main reasons why patients decide to have their lens removed from the eye, followed by an artificial lens implantation. In the case of lens opacification, which is additionally accompanied by refractive errors, the patient, thanks to the use of an appropriate intraocular lens model, gets rid of not only cataract, but also lower order aberrations resulting from the presence of accompanying eye defects: myopia, hyperopia, astigmatism or mixed defects [1]. The same benefit is obtained by patients who decide to have their own crystalline lens removed due to an existing refractive error. The key to obtaining the best postoperative effect is proper qualification for surgery. Removal of the lens from the eye, followed by subsequent implantation of an artificial lens can cause unwanted visual disturbances, generally referred to as higher order aberrations. Their appearance is influenced not only by the complex structure of the artificial lens, but also the structure of the eye [2]. A physician deciding to implant a lens with a complex structure (premium class lens) into the eye with a large pupil must consider a high risk of complaints on the part of the patient about unacceptable quality of vision, intensifying in scotopic conditions. The use of a monofocal lens significantly reduces the risk of adverse consequences in terms of the quality of vision, and additionally "protects" the physician, because nothing simpler can be implanted into the eye [3]. This is not a good option for a patient who expects greater benefits after cataract removal, and even worse for a patient who wants to get rid of an unwanted refractive error of the eye. Apart from a healthy cornea and retina, the elements to which special attention should be paid in the qualification process include: the position of the pupil centre in relation to the visual axis, i.e. angle kappa and the value of angle alpha formed between the visual axis and the eyeball positioning axis [4,5]. Another extremely important element is the size of the pupil and its proper reaction to light [6]. A review of the medical history is necessary because it provides further valuable information on the patient's functioning and post-treatment expectations.

In the process of qualifying for implantation of premium class lenses, bifocal, trifocal or multifocal lenses, patients with a pupil larger than $5.0 \mathrm{~mm}$ and with high values of angles alpha or kappa above $0.5 \mathrm{~mm}$, are most often disqualified due to the high risk of higher order aberrations that adversely affect the level of post-treatment satisfaction. It is also worth remembering that the presence of large pupils is most often associated with the predominance of the parasympathetic system in the body [7]. This in turn is associated with increased irritability, nervousness or meticulousness of the patient. This element may have a significant impact on the postoperative assessment of the quality of vision.

A high value of angle alpha is often observed in people with hyperopia, and this is quite a large group of patients who want to get rid of their refractive errors. For such more demanding 
patients with the above "ailments", the introduction of EDOF (Extended Depth Of Focus) lenses on the market seems to be an attractive offer [8]. Among products with such a structure, two lens models can be distinguished, i.e. evolve by Soleko and Lucidis by SAV IOL (Swiss Advanced Vision Intraocular Lens). Their common feature is a similar near addition, at the level of +2.75 Dsh (Evolve) and +3.0Dsph (Lucidis) $[9,10]$. Such an addition and the use of the physiological effect of reducing pupil size when focusing on close objects allows for easy reading and smooth transitions in vision from near to intermediate distances. Since the EDOF structure covers only 1.0-1.5 mm of the lens centre, the remaining part allows for good quality of vision to far distances to be obtained, characteristic of monofocal lenses [9,10].

\section{Objective}

Assessment of visual acuity to the far and near distances as well as the quality of vision in patients undergoing cataract surgery or Refractive Lens Exchange (RLE) due to an existing refractive error. In the study group, two models of lenses with a typical "pure" EDOF structure correcting a spherical refractive error were used: Evolve by Soleko and Lucidis by SAV IOL (Swiss Advanced Vision Intraocular Lens). In the process of qualifying for surgery, patients with a large pupil, above $5.0 \mathrm{~mm}$ and with large angles kappa and alpha, above $0.5 \mathrm{~mm}$, were considered. The above parameters were obtained preoperatively in an examination conducted using iTrace technology.

\section{Material and Methods}

36 patients were qualified for cataract removal or RLE procedures. Standard examinations were performed preoperatively, including best-corrected and uncorrected visual acuity too far and near distances, Intraocular Pressure Measurement (IOP), assessment of the anterior and posterior segment of the eye (after pupil dilation). In each case, special attention was paid to the function and size of the pupil (under photopic conditions), the physiology of which should be fully preserved. In addition, using iTrace technology, the condition of the cornea was analysed for the presence of higher order aberrations. Angles kappa and alpha were measured three times, and then the obtained values were averaged. The assessment of eye dominance is also a constant, important element of qualification for surgery. Only patients who lost their accommodative abilities were qualified for RLE.

\section{Results}

The lens was implanted into the capsular bag in each case undergoing surgery. 


\begin{tabular}{|c|c|}
\hline Value & Number \\
\hline Number of eyes & 43 \\
\hline Number of patients & 36 \\
\hline Lens implantation in both eyes & 7 \\
\hline Number of Evolve lenses & 29 \\
\hline Number of Lucidis lenses & 14 \\
\hline Cataract (eyes) & $24(55.8 \%)$ \\
\hline RLE (eyes) & $19(44.2 \%)$ \\
\hline Hyperopia before surgery (eyes) & 24 \\
\hline Myopia (eyes) & 5 \\
\hline Pre-surgery angle kappa $(\mathrm{mm})$ cataract/RWS & $\begin{array}{l}0.468 \pm 0.233 \\
0.368 \pm 0.319\end{array}$ \\
\hline Pre-surgery angle alpha $(\mathrm{mm})$ cataract/RWS & $\begin{array}{l}0.491 \pm 0.262 \\
0.484 \pm 0.143\end{array}$ \\
\hline $\begin{array}{l}\text { Pupil size }(\mathrm{mm}) \\
\text { before/after surgery - cataract }\end{array}$ & $4.85 / 4.17$ \\
\hline $\begin{array}{l}\text { Pupil size (mm) } \\
\text { before/after surgery - RLE }\end{array}$ & $5.06 / 4.84$ \\
\hline Average age (years) & $54 \pm 26$ \\
\hline No of Lucidis-RLE & 4 \\
\hline No of Evolve - RLE & 18 \\
\hline No of Lucidis - cataract & 10 \\
\hline No of Evolve - cataract & 11 \\
\hline
\end{tabular}

Table 1: Presents the obtained statistical values related to surgery. 
Almost $56 \%$ of patients were diagnosed with cataract, which indicates a large (44\%) proportion of patients who decided to undergo surgery due to an unwanted refractive error. In 29 patients, the EDOF lens was implanted monocularly.

The other eye, most often the dominant one, was implanted with a spherical or toric lens.

The product of choice was Mplus Comfort - a spherical or toric model by Oculentis, with an addition of $+1.5 \mathrm{Dsph}$, which gives the ability to see well also to intermediate distances. In the case of RLE, the largest group were patients with hyperopia in the range from +1.0 to +5.5 dioptres.

Statistical analysis: The results were analysed in terms of possible correlations between preand postoperative visual acuity, the reason for surgery, the size of angles kappa and alpha, and the pupil size.

In the case of studying the correlation between two parameters, the formula for the correlation coefficient was used, while when comparing the data, the mean value of the parameter studied was used.

The results of the studied correlations, presented in the form of graphs:

1. The correlation between the preoperative angle kappa value $(\mathrm{mm})$ and postoperative Uncorrected Visual Acuity to far distance (UCVA) using Lucidis lens and Evolve lens (Fig. 1)

Results for both lenses: Weak positive correlation.

Interpretation: The higher the angle kappa, the higher the logMar value and therefore the poorer visual acuity to far distances.

2. The correlation between the preoperative angle alpha $(\mathrm{mm})$ value and postoperative Uncorrected Visual Acuity to far distances (UCVA) surgery using Lucidis lens and Evolve lens (Fig. 2)

Results: Moderate positive correlation for Lucidis, weak negative correlation for evolve.

Interpretation: a higher angle alpha has a moderate negative effect on visual acuity to far distances in the case of Lucidis lens implantation, in the case of the Evolve model, increasing the angle does not affect the deterioration of vision to far distances.

3. The correlation between the pupil size $(\mathrm{mm})$ before surgery and postoperative Uncorrected Visual Acuity to far distances (UCVA), using Lucidis lens and Evolve lens (Fig. 3)

Results: Weak negative correlation for both lenses.

Interpretation: An increase in pupil size may have a weak impact on the decrease in LogMar values, i.e. improvement of vision to far distances. 
4. The correlation between the pupil size $(\mathrm{mm})$ and Uncorrected Visual Acuity to near distances (UCVA) after surgery, measured in Snellen charts in the eye with the Evolve lens (Fig. 4)

Results: Weak positive correlation for evolve.

Interpretation: An increase in pupil size may have a weak impact on the increase in Snellen value, i.e. deterioration of vision to near distances.
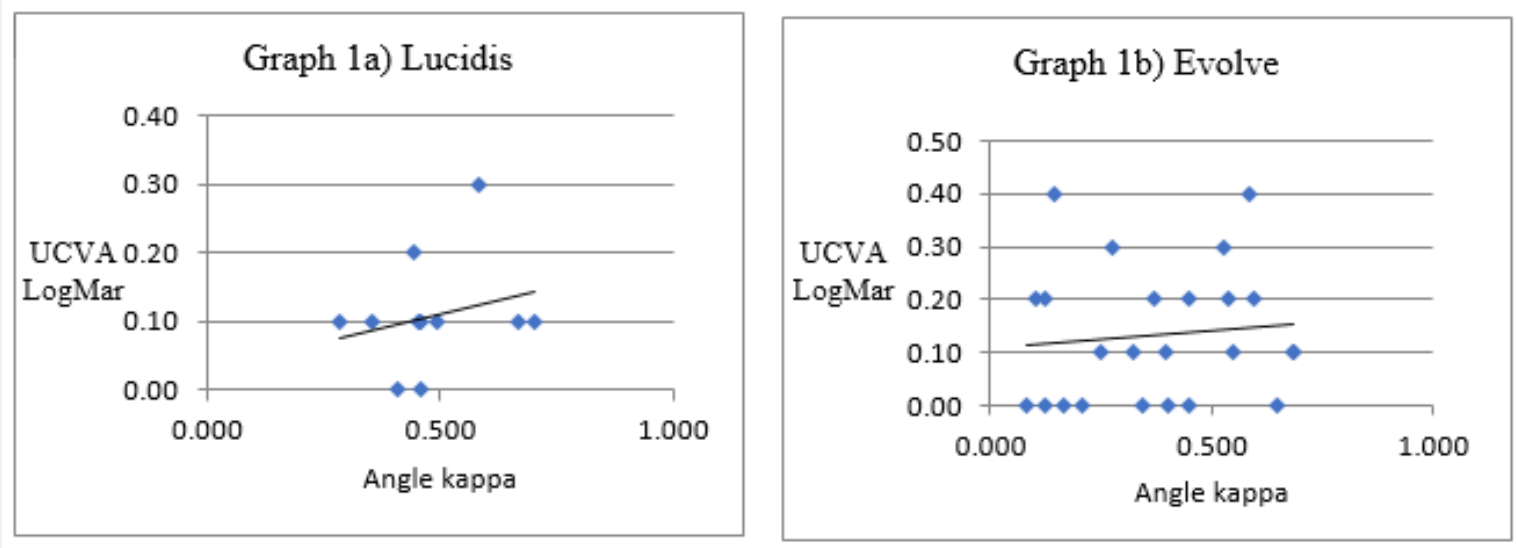

Figure 1: Correlation between the preoperative angle kappa value $(\mathrm{mm})$ and postoperative Uncorrected Visual Acuity to far distance (UCVA) using Lucidis lens, and 1b, using Evolve lens.

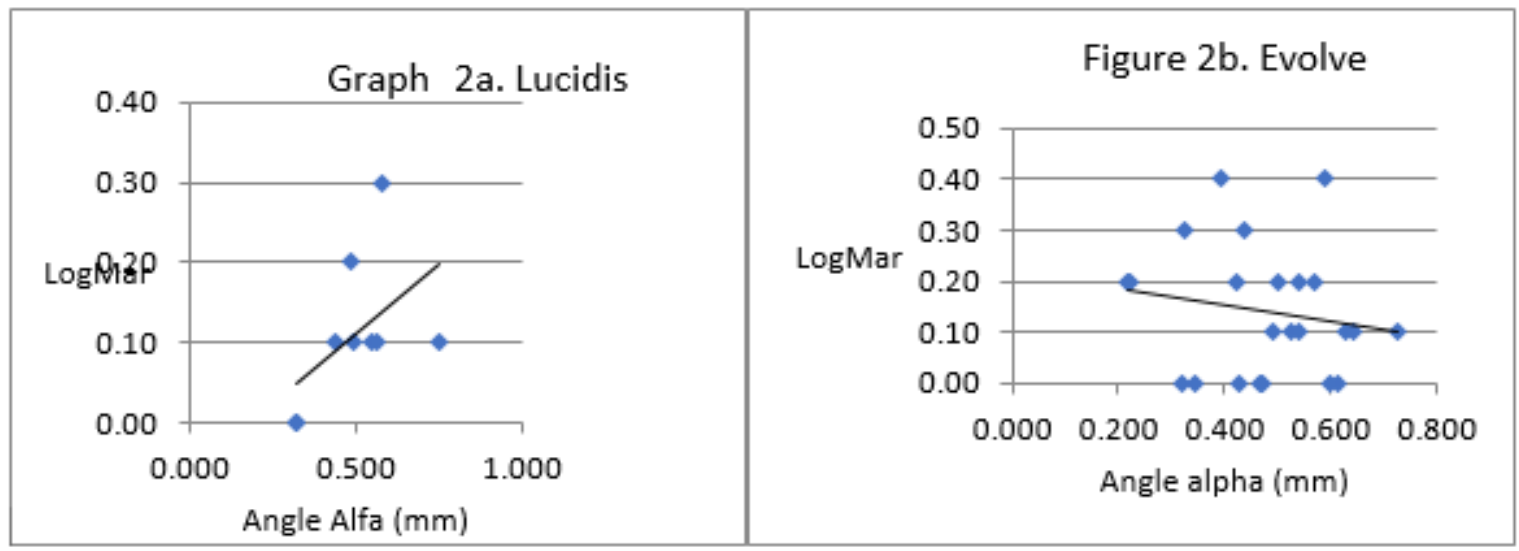

Figure 2: Correlation between the preoperative angle alpha $(\mathrm{mm})$ value and postoperative Uncorrected Visual Acuity to far distance (UCVA) using Lucidis lens and 2b, using Evolve lens. 

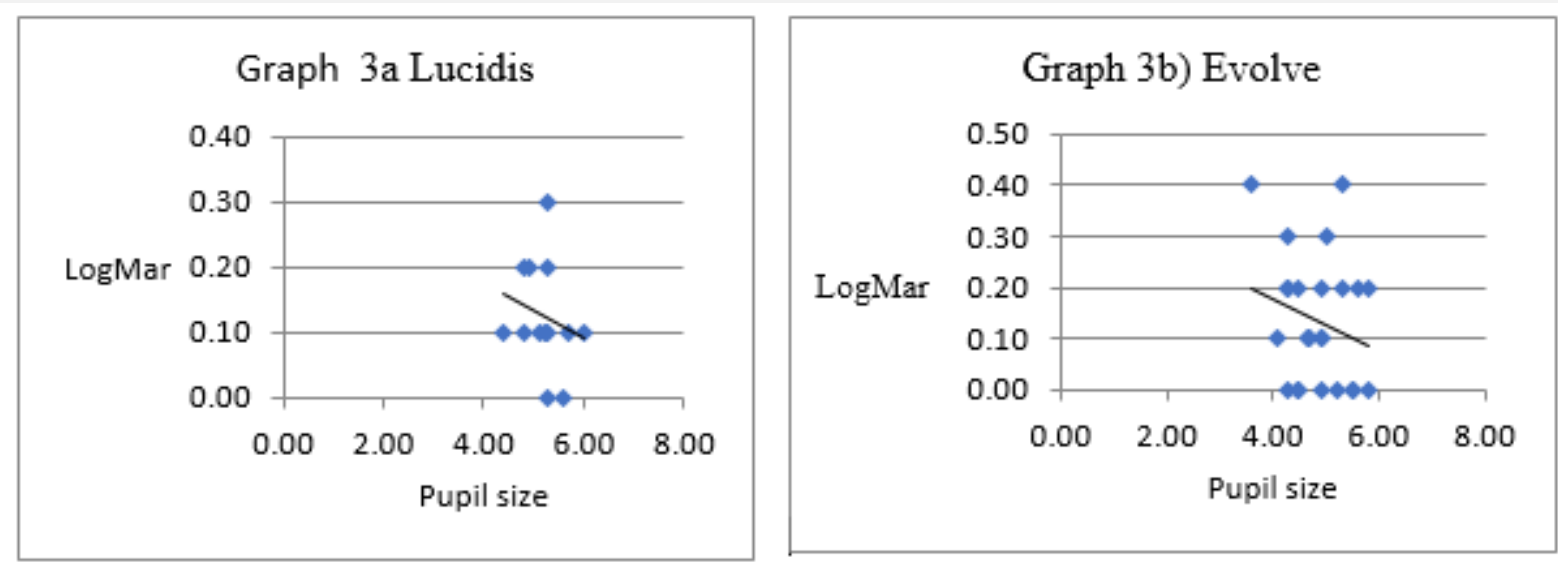

Figure 3: Correlation between the pupil size $(\mathrm{mm})$ measured before surgery and postoperative, Uncorrected Visual Acuity to far distance (UCVA), using Lucidis lens and 3b, using Evolve lens.

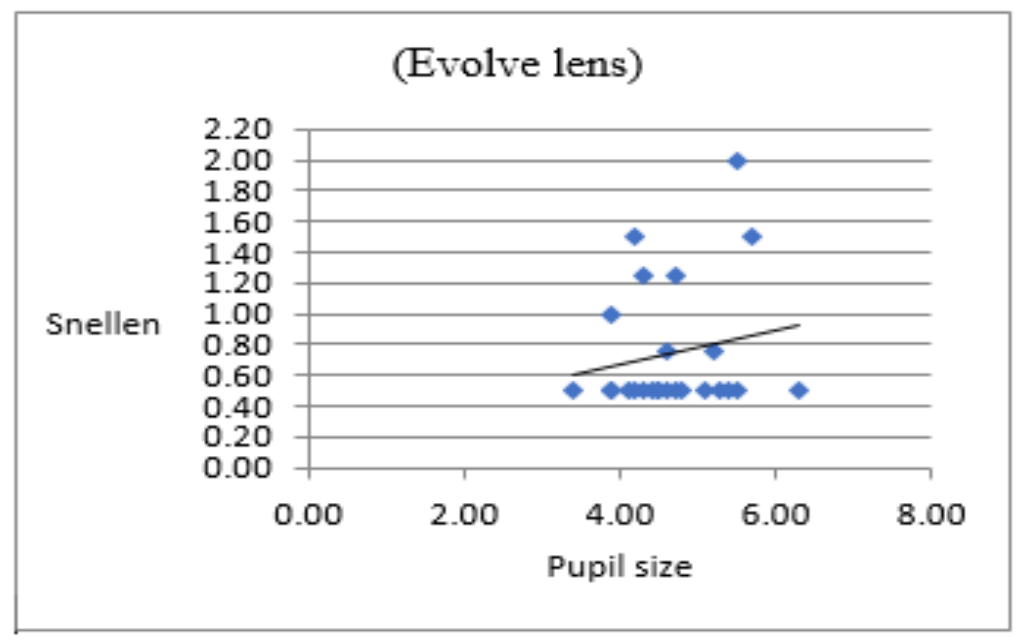

Figure 4: Correlation between the pupil size $(\mathrm{mm})$ and uncorrected, postoperative visual acuity to near distance (UCVA), measured in Snellen charts in the eye with the Evolve lens. 
UCPVA

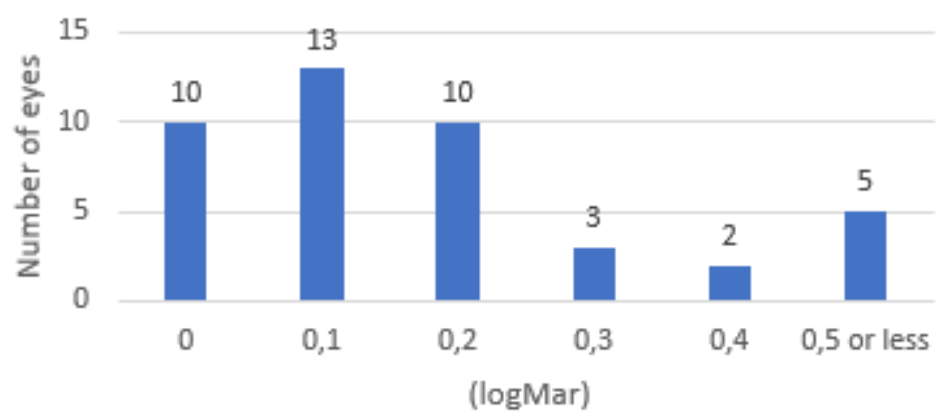

Figure 5: Postoperative values of Uncorrected Visual Acuity to far distance (UCPVA), obtained for the entire group.

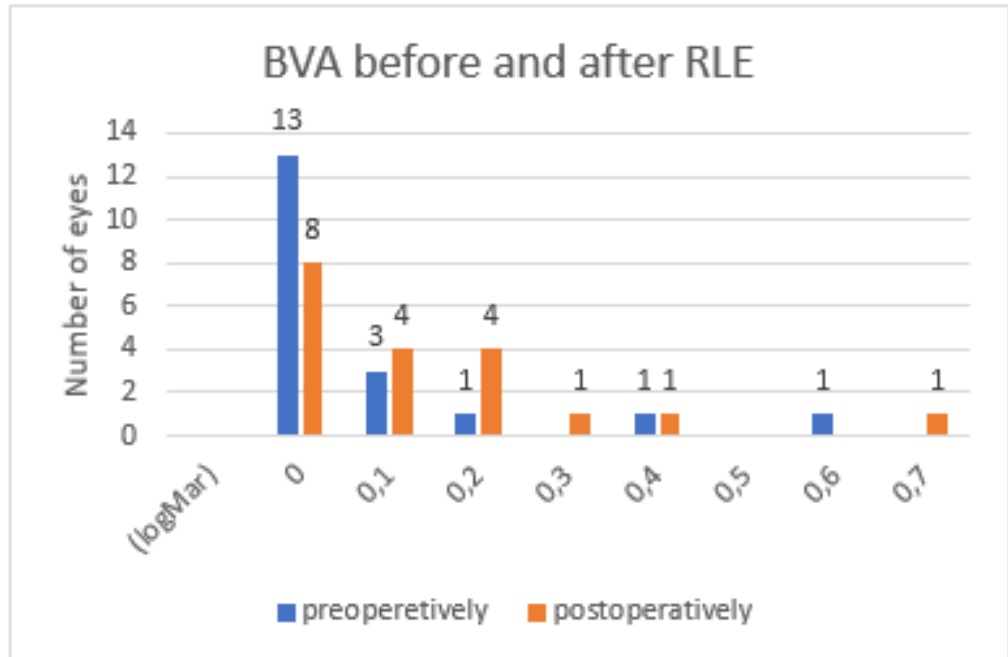

Figure 6: Comparison of Best-Corrected Visual Acuity to far distance (BCVA) in the RLE group achieved preoperatively, with uncorrected visual acuity measured postoperatively.

\section{Visual Acuity}

LogMar charts were used to assess visual acuity to far distances, and Snellen charts to assess visual acuity to near distances.

Patients with visual acuity to far distances better than 0.5 (logMar) were analysed statistically.

A comparative analysis of the values obtained before and one month after surgery was performed. 
The mean values of visual acuity to far distances, best corrected, obtained before surgery were 0.26 (in the range from 0.0 to 2.0), and after surgery, uncorrected, 0.27 (in the range from 0.0 up to 0.6 ). The mean value of uncorrected visual acuity to near distances, after surgery was $0.65 \pm 0.15$.

The obtained postoperative values of Uncorrected Visual Acuity to far distances (UCPVA) for the entire group are presented in the form of Fig. 5.

\section{Refractive Lens Replacement and Visual Acuity to Far Distances}

A comparison of Best-Corrected Visual Acuity (BCVA) in the RLE group achieved preoperatively with uncorrected visual acuity measured postoperatively is presented in the form of Fig. 6.

The mean values of the best visual acuity to far distances in the group with RWS reached the preoperative value of 0.05 , and the postoperative value of 0.11 . Slightly better values were obtained in eyes with Lucidis lens implants, reaching 0.1 before surgery and 0.07 after surgery. In the eyes with the Evolve lens, the decrease in postoperative visual acuity was more noticeable ( 0.04 before, 0.11 after). However, it is difficult to write about proper statistics here, because the number of Lucidis lens implants within the RWS group included only 4 eyes.

The reasons why visual acuity to far distances after surgery did not reach a value better than $0.5 \log$ Mar included cases with geographic atrophy, partially involving the fovea, the condition after eye injury followed by pupillary sphincter dysfunction and very narrow pupils, reaching values of 2.8-3.3 mm after cataract removal, despite their preoperative parameters at the level of $3.8-4.3 \mathrm{~mm}$.

\section{Subjective Assessment of the Quality of Vision by the Patient}

No halo and glare symptoms have been reported. Some patients reported negative photopsia. Deterioration of visual acuity to far distances in the presence of high light intensity is the main complaint of patients in the initial postoperative period. These symptoms intensified in patients who underwent RWS in a hyperopic, dominant eye, where the postoperative pupil size was smaller than $3.8 \mathrm{~mm}$. Persistent reduced values of visual acuity to far distances occurred when the pupil size was smaller than $3.0 \mathrm{~mm}$.

Wearing sunglasses or reducing light intensity resulted in an almost immediate improvement in vision.

Conversely, the loss of the ability to read freely was noticed by most of the patients in the conditions of significantly lower light intensity, especially when the EDOF lens was implanted monocularly. 
For one patient with a preoperative pupil size of $6.5 \mathrm{~mm}$, visual acuity to near distances after implantation of the Evolve model was D-2.25. Only in conditions of significant sunlight, the patient was able to read the text with the size D-0.5.

The pupil size in the whole group, before surgery, ranged from 3.7 to $6.5 \mathrm{~mm}$, after surgery from 2.9 to $6.0 \mathrm{~mm}$.

\section{Discussion}

Can a "pure" EDOF lens be offered to every patient? No. Analysing the results obtained and the complaints reported by patients, a small pupil is the main contraindication to the use of this lens. Its low values correlate with poorer visual acuity to far distances. Conversely, enlarging the pupil size improves vision to far distances. In the case of the studied group, in the process of qualifying for implantation, mainly eyes with medium-size and large pupils were selected. The first experiments showed that the small pupil with values below $3.5 \mathrm{~mm}$ before surgery resulted in poor values of visual acuity to far distances, especially in eyes with diagnosed cataract, in which the average postoperative reduction in pupil size was $0.68 \mathrm{~mm}$.

Concerning vision to near distances, the situation is quite the opposite. This can be explained by the varying contribution to vision of the individual lens elements. In the case of a pupil with a size of $3 \mathrm{~mm}$, the light distribution is $2: 1$, which means that statistically $66 \%$ of light passes through the monofocal part of the lens when looking to far distances. If the pupil size is $5 \mathrm{~mm}$, the light distribution is $4: 1$, theoretically resulting in light distribution to far distances through $80 \%$ of the lens surface.

EDOF lenses are a typical product, the use of which depends on pupil size and light intensity. As shown by the results, in the RLE group, visual acuity to far distances was slightly worse after surgery than the one obtained in best correction before surgery, and it additionally deteriorated in conditions such as significant sun exposure.

This information is extremely important. For this reason, a patient who undergoes RLE in the dominant eye because of preoperative hyperopia may experience deterioration rather than improvement of visual acuity to far distances. Therefore, it is not recommended. The dominant eye should have some correction allowing for predictable quality of vision to far distances to be obtained.

Predictable values of vision to far distances with pure EDOF lenses can be obtained in eyes where pupil size is unlikely to be smaller than $4.0 \mathrm{~mm}$ or less. Preoperative values above 5 $5.5 \mathrm{~mm}$ allow us to obtain this certainty.

What positively distinguishes lenses with a pure EDOF structure in comparison to other premium class models is the possibility of using them in eyes with high values of angles kappa and alpha. 
In the studied group, the values of angle alpha reached 0.7 , of angle kappa $0.6 \mathrm{~mm}$, without causing adverse symptoms, both in photo and scotopic conditions. Statistical analysis showed a weak (Evolve) or moderate (Lucidis) correlation between increasing angles kappa and alpha and a slight decrease in visual acuity in eyes with implants of both lens models. Only in the Evolve lens group the increase in angle alpha did not cause deterioration of vision to far distances.

In the case of monocular implantation, EDOF lenses were mainly implanted into the nondominant eye, thus obtaining a favourable effect of vision to far and near distances. A model with a smaller addition (+1.5 Dsph) from another company (Oculentis) was most often implanted into the dominant eye enabling vision up to intermediate distances, which is less dependent on pupil size.

The use of premium class lenses with a more complex structure is associated with the risk of unfavourable visual disturbances, especially in hyperopic eyes, where angle alpha values can be significant. In these cases, the use of a "pure" EDOF lens gives the patient more benefits than a monofocal lens can provide.

In the case of a very large pupil, with values above $6 \mathrm{~mm}$, diagnosed in both eyes, the implantation of the above models of lenses allows us to obtain the quality of vision to far distances comparable to the quality of vision obtained with a monofocal lens. In the case of vision to near distances, light intensity must be very good for the pupil to be properly narrowed, allowing the central EDOF structure to be used for reading. It is worth noting that the correct pupil function is required if you want to use EDOF models.

\section{Conclusion}

The use of "pure" EDOF lenses should be avoided in eyes with a very small pupil, in particular if you want to correct the dominant eye with preoperative hyperopia. However, such a lens structure allows for the correction of eyes with a large, preoperative angles kappa and alpha, and eyes with a large pupil without significantly affecting the postoperative quality of vision and visual acuity. This is the advantage of lenses with a "pure" EDOF structure over premium class lenses with a more complex structure. Their use makes it possible to obtain vision comparable in quality to correction with the use of monofocal lenses. Their advantage over monofocal lenses involves vision to near distances without the need for additional correction. If you wish to perform correction in both eyes in patients with high values of angles kappa or alpha, the preoperative pupil size should not be less than $4.8-5.0 \mathrm{~mm}$ and it is better to surgically correct the non-dominant eye first. 


\section{References}

1. Lee CY, Huang JY, Sun CC, Yang SF, Chen HC, Lin HY. Correlation and predictability of ocular aberrations and the visual outcome after quadrifocal intraocular lens implantation: a retrospective longitudinal study. BMC Ophthalmol. 2019;19(1):1-8.

2. Aberrations of the eye. Wikipedia. [Last accessed on: June 15, 2021] https://en.wikipedia.org/wiki/Aberrations_of the eye

3. Kim CY, Chung SH, Kim TI, Cho YJ, Yoon G, Seo KY. Comparison of higher-order aberration and contrast sensitivity in monofocal and multifocal intraocular lenses. Yonsei Med J. 2007;48(4):627-33.

4. Piracha A. Using angle alpha in premium IOL screening. Cataract Surgery Feature Stories. 2016.

5. Tipperman R. Is this IOL centered? Angle kappa and multifocal IOLs. Eyeworld. 2014.

6. Artigas JM, Menezo JL, Peris C, Felipe A, Díaz-Llopis M. Image quality with multifocal intraocular lenses and the effect of pupil size: comparison of refractive and hybrid refractive-diffractive designs. J Cataract Refract Surg. 2007;33(12):2111-7.

7. Schumann A, Kietzer S, Ebel J, Bär KJ. Sympathetic and parasympathetic modulation of pupillary unrest. Front Neurosci. 2020;14:178.

8. Fuller D. Extended depth-of-focus optics: a guide for optometrists. Rev Optometry. 2019

9. Kanclerz P, Toto F, Grzybowski A, Alio JL. Extended depth-of-field intraocular lenses: an update. Asiapacific J Ophthalmol. 2020;9(3):194-202.

10. Sieburth R, Chen M. Intraocular lens correction of presbyopia. Taiwan J Ophthalmol. 2019;9(1):4. 